\title{
An interesting plane dendroid
}

by

David P. Bellamy * (Newark, Del.)

Abstract. Using inverse limits and geometric techniques, a dendroid $M$ is constructed in the plane with connected set of endpoints, and such that the endpoints and only the endpoints of $M$ are arcwise accessible from the complement of $M$.

I. Introduction. In this paper, a plane dendroid $M$ is constructed such that only its endpoints, in the sense of Lelek [3], are arcwise accessible from the complement of $M$. Further, the set $E$ of endpoints of $M$ is a connected set and has a "circle-like" property, namely, that while no point of $E$ separates $E$, each pair of points of $E$ does separate $E$. $E$ must be a dense subset of $M$, so that the closure of $E$ does not separate the plane. When this example was presented in Professor Borsuk's seminar, the following two questions were raised:

1 (Krasinkiewicz). If a plane dendroid has the property that its endpoints are its only arcwise accessible points, must the set of endpoints be connected?

2 (Borsuk). Is $M$ irreducible with respect to the property that its endpoints and its accessible points are the same?

In the last section, examples are described giving a negative answer to each of these questions.

I would like to thank J. Krasinkiewicz and P. Minc for many helpful conversations during the preparation of this paper. The questions leading to this work arose during the seminar on metric continua led by Krasinkiewicz and Minc at Instytut Matematyczny, PAN in Warszawa in the fall of 1975.

The following terminology will be used. The arc in a given dendroid from $p$ to $q$ will be denoted $[p, q] . \mathrm{Cl}(A)$ denotes the closure of a set $A$, in whatever space it is being considered. $E^{2}$ is the real Euclidean plane and $d$ its usual metric. If $A \subseteq E^{2}$, $H(A)$ is the convex hull of $A$. A $V$-arc is an arc in $E^{2}$ which is the union of two noncollinear straight segments with a common endpoint. The interior of a $V$-arc $V$,

* Except for the second example in the last section and the final preparation of the manuscript, this research was done while the author was working at the Institute of Mathematics of script, this research was done while the author was working at the Institute of Mathematics of
the Polish Academy of Sciences as a participant in an exchange program sponsored jointly by the Polish Academy of Sciences, Warsaw, and the National Academy of Sciences, Washington, D. C. 
denoted $I(V)$, means the interior of $H(V)$. The term vertex of a $V$-arc is self-explanatory. For three non-collinear points, $p, q$, and $r$ in $E^{2}, V(p, q, r)$ denotes the $V$-arc with $p$ and $r$ as opposite endpoints and $q$ as vertex. The boundary of a $V$-arc $V(p, q, r)$, denoted $\operatorname{Bd}(V(p, q, r))$ is the straight segment from $p$ to $r$ and $\operatorname{bd}(V(p, q, r))$ is $\operatorname{Bd}(V(p, q, r))$ with its endpoints deleted. (Thus $H(V)$ is the disjoint union of $V$, $I(V)$, and bd(V).)

If $K$ is a continuum in $E^{2}$ and $p \in K$, we shall say briefly that $p$ is "accessible in $K^{\prime \prime}$ to mean that $p$ is arcwise accessible from $E^{2}-K$.

The next definition is rather technical. An exposed $V$-arc in a dendroid $K \subseteq E^{2}$ will mean a $V$-arc $V$ such that: 1) $V \subseteq K$;2) $K \cap H(V)=V$; 3) The endpoints of $V$ are also endpoints of $K$; and 4) The nonendpoints of $V$ are not accessible in $K \cup H(V)$. (It is an immediate consequence of 2) that each point of an exposed $V$ arc $V$ is accessible in $K$, by a segment from any point in $I(V)$. Thus, 4) could be restated: The nonendpoints of $V$ are accessible in $K$ only by meens of arcs which meet $I(V)$.) A $V$-arc $V$ which satisfies all of the above except that its vertex may be accessible in $K \cup H(V)$ will be called a $v$-exposed $V$-arc in $K$.

In an inverse sequence $\left\{X_{i} ; h_{i}\right\}_{i=1}^{\infty}, h[k, j]: X_{k} \rightarrow X_{j}$ denotes the appropriate multi-step bonding map. $X$ is the inverse limit of the sequence. (Similarly for any other letter in place of $X$.) If $K \subseteq X, K_{i}$ is the image of $K$ under the projection of $X$ to $X_{i}$.

The construction proceeds as follows: First we shall construct a dendroid $B$ which is our "building block". We shall then construct an inverse sequence $\left\{M_{i} ; h_{i}\right\}_{i=1}^{\infty}$, where each $M_{i}$ is built up out of specially constructecl copies of $B$. Then we shall prove that $M$ embeds in the plane, using a theorem of Anderson and Choquet to be restated below in a convenient form, and that $M$ is a dendroid with the desired properties.

The following lemmas will smooth the way somewhat.

LEMMA 1. Any inverse limit of dendroids with monotone bonding maps is a dendroid.

Proof. Let $\left\{M_{i} ; h_{i}\right\}_{i=1}^{\infty}$ be such an inverse sequence, and let $\left\langle p_{i}\right\rangle_{i=1}^{\infty}$, $\left\langle q_{i}\right\rangle_{i=1}^{\infty} \in M$. Since $h_{i}$ is monotone and $M_{i}$ is hereditarily unicoherent for each $i$, it is easily verified that $h_{i}$ maps $\left[p_{i+1}, q_{i+1}\right]$ monotonically onto $\left[p_{l}, q_{i}\right]$. Thus, the inverse limit, $A$, of this sequence of arcs is an arc in $M$ from $\left\langle p_{i}\right\rangle_{i=1}^{\infty}$ to $\left\langle q_{i}\right\rangle_{i=1}^{\infty}$. If $W \subseteq M$ is any continuum containing these two points, $\left[p_{l}, q_{i}\right] \subseteq W_{i}$ for each $i$, so that $A \subseteq W$. Thus $M$ is arcwise connected and hereditarily unicoherent and the proof is done.

In an inverse sequence $\left\{X_{i} ; h_{i}\right\}_{i=1}^{\infty}$ where all the bonding maps are retractions there is a natural embedding $k_{i}: X_{i} \rightarrow X$ defined by: $k_{i}(x)$ is that sequence $\left\langle x_{j}\right\rangle_{j=1}^{\infty}$ such that $x_{j}=x$ for all $j \geqslant i$. (For $j<i, x_{j}$ is determined by the bonding maps.) If $e_{i j}: X_{i} \rightarrow X_{j}$ is the inclusion map for $i \leqslant j$, then $k_{i}=k_{j}$ o $e_{i j}$. Henceforth in such an inverse system each $X_{j}$ will be identified with its image under $k_{j}$ to avoid cumbersome notation, and $X_{\infty} \subseteq X$ will be defined by $X_{\infty}=\bigcup_{n=1}^{\infty} X_{n}$.
LEMMA 2. Let $\left\{X_{i} ; h_{i}\right\}_{i=1}^{\infty}$ be an inverse sequence of dendroids and monotone retractions. Then each point of $X-X_{\infty}$ is an endpoint of $X$.

Proof. Let $H_{i}: X \rightarrow X_{i}$ be the projection. Each $H_{i}$ is also a monotone retraction. First observe that $X-X_{\infty}$ contains no arc, for if $[p, q] \subseteq X-X_{\infty}$ were an arc, then there would be an $n$ such that $H_{n}(p) \neq H_{n}(q)$. Then

$$
[p, q] \cup H_{n}^{-1}\left(H_{n}\{p, q\}\right) \cup H_{n}[p, q]
$$

would be a nonunicoherent continuum in $X$ since

$$
\left([p, q] \cup H_{n}^{-1}\left(H_{n}\{p, q\}\right)\right) \cap H_{n}[p, q]=\left\{H_{n}(p), H_{n}(q)\right\} .
$$

Next, if $[p, q]$ were an arc with a point $b \in[p, q]-\{p, q\}$ such that $b \in X-X_{\infty}$, then since $X-X_{\infty}$ contains no arc, there would be points $r \in[p, b]$ and $s \in[b, q]$ such that $r, s \in X_{\infty}$. Thus, for some $k ; r, s \in X_{k}$. But then $X_{k} \cup[r, s]$ would be a nonunicoherent continuum since $X_{k} \cap[r, s]$ contains boib $r$ and $s$ but not $b$. Therefore an arc in $X$ can meet $X-X_{\infty}$ only at its endpoints, completing the proof.

LEMMA 3. If $S$ is a complete metric space and $\left\{M_{i}\right\}_{i=1}^{\infty}$ is an inverse sequence of compact subsets of $S$ and monotone retractions, and if for each $\varepsilon>0$ there exists an $n$ such that for each $p \in M_{n}$,

$$
\operatorname{dia}\left[\bigcup_{i=n}^{\infty}(h[i, n])^{-1}(p)\right]<\varepsilon
$$

and if for each $n$ the family $\{h[i, n]\}_{i=n}^{\infty}$ is uniformly equicontinuous, then $H: M \rightarrow S$, defined by $H\left[\left\langle p_{i}\right\rangle_{i=1}^{\infty}\right]=\lim _{i \rightarrow \infty} p_{i}$ is a homeomorphism of $M$ onto $\mathrm{Cl}\left[\bigcup_{i=1}^{\infty} M_{i}\right]$. Furthermore (with the identifications made above before Lemma 2), $H \mid M_{t}$ is the identity on $M_{i}$.

Proof. Except for the last sentence, this is a special case of Theorem 1 of $[1$, p. 348]. The last sentence is clear.

II. Construction of the example. Let $X$ denote the Cantor set consisting of all those numbers in $[0,1]$ which can be expanded base 5 using only the digits 0,2 , and 4 . (This set may be obtained by dividing the interval into fifths and removing the second and fourth fifths; that is, the open intervals $\left(\frac{1}{5}, \frac{2}{5}\right)$ and $\left(\frac{3}{5}, \frac{4}{5}\right)$ and then recursively repeating this process on the remaining intervals at each step.) Observe that $\frac{1}{2} \in X$ and that $\frac{1}{2}$ is not an endpoint of a deleted interval.

Then there is a map $\mu: X \rightarrow[0,1]$ defined by

$$
\mu\left[\sum_{k=1}^{\infty} a_{k} \cdot 5^{-k}\right]=\sum_{k=1}^{\infty}\left[\frac{1}{2} a_{k}\right] \cdot 3^{-k},
$$

where it is understood that each $a_{k}$ is even. The properties of $\mu$ are similar to those of the Cantor ternary function. The facts we need about $\mu$ are: 1) $\mu$ is surjective; 2) $\mu$ has a continuous extension $\bar{\mu}:[0,1] \rightarrow[0,1]$ which is constant on each of the 
components of $[0,1]-X ; 3)$ If $x$ is an endpoint of one of the intervals deleted in the construction of $X$, then $\mu(x)$ is a triadic rational; and 4) $\mu(x)=x$ if $x \in\left\{0, \frac{1}{2}, 1\right\}$

First consider a copy, $B_{0}$, of the mapping cylinder of $\mu$, embedded in $E^{2}$ as follows: $B_{0}$ is the union of all straight line segments from $(x, 0)$ to $(\mu(x), 1)$ for $x \in X$ (see Fig. 1.)

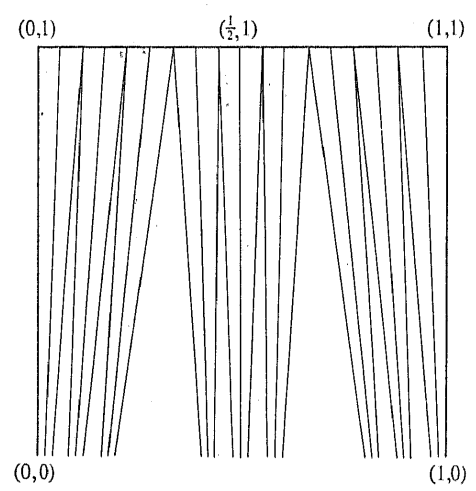

Fig. 1. $B_{0}$

$B_{0}$ is a dendroid and has a $v$-exposed $V$-arc corresponding to each deleted interval in the construction of $X$. For technical reasons, define also a slightly different dendroid, $C_{0}$, as follows: $C_{0}$ is the union of the two sets

and

$$
\left\{\left(x+\frac{1}{2}, y\right) \mid(x, y) \in B_{0} \text { and } x \leqslant \frac{1}{2}\right\}
$$

$$
\left\{\left(x-\frac{1}{2}, y\right) \mid(x, y) \in B_{0} \text { and } x \geqslant \frac{1}{2}\right\} \text {. }
$$

Geometrically, we have cut $B_{0}$ in two along the vertical line segment at $x=\frac{1}{2}$ and sewn it back together, identifying the vertical segments at $x=0$ and $x=1$ together. $C_{0}$ is a dendroid since $B_{0}$ contains the vertical segment from $(x, 0)$ to $(x, 1)$ for $x=0, \frac{1}{2}$, or 1 . The important thing to observe about $C_{0}$ and $B_{0}$ here is that the vertices of the $v$-exposed $V$-arcs in $C_{0}$ have $x$-coordinates of the form $a \pm \frac{1}{2}$, where $a$ is a triadic rational, while those of $B_{0}$ are themselves triadic rationals. Hence no vertex of one of these $V$-arcs in $B_{0}$ is a vertex of one in $C_{0}$. This last statement will also hold for $B_{1}$ and $C_{1}$, for $B$ and $C$, and finally for $B$ and $\bar{C}$, all to be constructed below.

The retraction $r_{0}: B_{0} \rightarrow[0,1] \times\{1\}$, defined, for $t \in[0,1]$ and $x \in X$, by $r_{0}(t(x, 0)+(1-t)(\mu(x), 1))=(\mu(x), 1)$

is just the map which sends each point on the line segment from $(x, 0)$ to $(\mu(x), 1)$ to the point $(\mu(x), 1)$. This is just the standard retraction of a mapping cylinder onto its range space. Observe that $r_{0}$ is a monotone retraction, as will be every map which we denote by the letter $r$ with or without subscripts or other means of distinction. The reader may check this in each case.

Let $D_{0}=[0,1] \times[0,1] . r_{0}$ has a continuous extension $\bar{r}_{0}: D_{0} \rightarrow[0,1] \times\{1\}$ defined by $\bar{r}_{0}(t(x, 0)+(1-t)(\bar{\mu}(x), 1))=(\bar{\mu}(x), 1)$, where now $t, x \in[0,1]$. $\bar{r}_{0}$ is constant on the convex hull of each $v$-exposed $V$-arc in $B_{0}$.

$B_{0}, C_{0}$, and $D_{0}$ have been constructed where they are easy to visualize; they will now be moved to where they are easier to work with. Let $f: E^{2} \rightarrow E^{2}$ be defined by $f(x, y)=(2 x-1, y-1)$, and let $B_{1}=f\left(B_{0}\right) ; C_{1}=f\left(C_{0}\right) ;$ and $D_{1}=f\left(D_{0}\right)$. The retractions $f \circ r_{0} \circ\left(f^{-1} \mid B_{1}\right)$ and $f \circ \bar{r}_{0} \circ\left(f^{-1} \mid D_{1}\right)$ will be called $r_{1}$ and $\bar{r}_{1}$, respectively.

$B, C$, and $D$ are now obtained from $B_{1}, C_{1}$, and $D_{1}$ by setting:

$$
\begin{aligned}
& B=\left\{(x, y) \in B_{1}|y \geqslant| x \mid-1\right\}, \\
& C=\left\{(x, y) \in C_{1}|y \geqslant| x \mid-1\right\}, \\
& D=\left\{(x, y) \in D_{1}|y \geqslant| x \mid-1\right\} .
\end{aligned}
$$

Geometrically, this means that $B$ (respectively $C, D$ ) is the set of points of $B_{1}$ (respectively $\left.C_{1}, D_{1}\right)$ on or above $V((-1,0),(0,-1),(1,0))$ (see Figure 3 or the lower half of Figure 2). The retractions $\bar{r}_{1} \mid D$ and $r_{1} \mid B$ will be called simply $\bar{r}$ and $r$, respectively. Again, $r$ is a restriction of $\bar{r}$, and the range of each is $[-1,1] \times\{0\}$, and $\vec{r}$ is constant on the convex hull of each of the $v$-exposed $V$-arcs of $B$.

Let $M_{1}$ denote the union of $B$ with the reflection of $C$ through the $x$-axis; that is, $M_{1}=B \cup \bar{C}$, where $\bar{C}=\{(x, y) \mid(x,-y) \in C\}$ (see Fig. 2). It is clear that $M_{1}$ and $B$ are dendroids. Observe also the following facts about $M_{1}$ and $B$.

(i) The accessible points of $M_{1}$ consist of its endpoints together with the points of countably many exposed $V$-arcs in $M_{1}$. (The $v$-exposed $V$-arcs of $B$ and of $\bar{C}$ are exposed $V$-arcs in $M_{1}$.)

(ii) The only points of $[-1,1] \times\{0\}$ which are accessible in $B$ by an arc lying entirely in the closed lower half-plane are the endpoints $(-1,0)$ and $(1,0)$ and the vertices of the $v$-exposed $V$-arcs in $B$. In particular, $(0,0)$ is not accessible by such an arc. $\left((0,0)\right.$ corresponds to $\left(\frac{1}{2}, 1\right)$ in $B_{0}$.)

The dendroids $M_{i}$ for $i \geqslant 2$ will be constructed as unions of $M_{1}$ with particular copies of $B$ used to "cover up" the exposed $V$-arcs of $M_{1}$. No further use will be made of $C$ apart from its place in $M_{1}$.

For $1 \geqslant \varepsilon>0$, let $B\langle\varepsilon\rangle$ (respectively $D\langle\varepsilon\rangle$ ) denote that portion of $B$ (respectively $D)$ on or above $V((-1,0),(0,-\varepsilon),(1,0))$; that is,

$$
B\langle\varepsilon\rangle=\{(x, y) \in B|y \geqslant| \varepsilon x \mid-\varepsilon\}
$$

and similarly for $D\langle\varepsilon\rangle$ (see Fig. 3).

Let $r\langle\varepsilon\rangle=r|B\langle\varepsilon\rangle ; \bar{r}\langle\varepsilon\rangle=\bar{r}| D\langle\varepsilon\rangle$. By choosing $\varepsilon$ sufficiently small, the maximum diameter of point inverses under $\bar{r}\langle\varepsilon\rangle$ and $r\langle\varepsilon\rangle$ can be made arbitrarily 


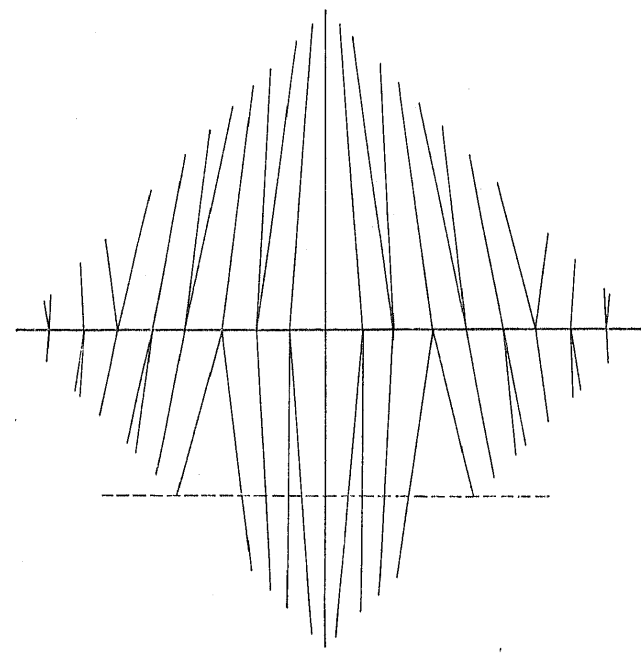

Fig. 2. $M_{1}\left(N_{1}\right.$ is the square indicated by the dashed lines.) The horizontal dashed line is used in Section VI

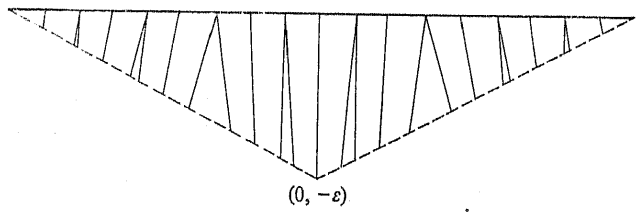

Fig. 3. $B\langle e\rangle$ is that portion of $B$ above the dashed $V$-arc

small. Next define, for each fourtuple $[a, b, c, d]$ of positive reals, the homeomorphism $g[a, b, c, d]$ of $E^{2}$ by:

$$
g[a, b, c, d](x, y)=\left\{\begin{array}{lll}
(a x, y+b x) & \text { if } & x \leqslant 0 \\
(c x, y-d x) & \text { if } & x \geqslant 0 .
\end{array}\right.
$$

Whenever convenient, $\alpha$ will denote a fourtuple of positive reals. Each $g[\alpha]$ is the identity on the $y$-axis and is linear in the left and right closed half planes, and bends both halves of the $x$-axis downward, to slopes of $b / a$ and $-d / c$ respectively. The image under $g[\alpha]$ of any $V$-arc $V$ which misses the $y$-axis is a $V$-arc, and $g[\alpha](I(V))=I(g[\alpha](V))$. In particular, this is true of the $v$-exposed $V$-arcs in each $B\langle\varepsilon\rangle$.
Denote by $V[\alpha]$ the $V$-arc $g[\alpha]([-1,1] \times\{0\})$. By appropriate choice of $\alpha$, $V[\alpha]$ can be made congruent with any preassigned $V$-arc in $E^{2}$.

Let $B[\alpha, \varepsilon]=g[\alpha](B\langle\varepsilon\rangle)$ and $D[\alpha, \varepsilon]=g[\alpha](D\langle\varepsilon\rangle)$, and define $r[\alpha, \varepsilon]: B[\alpha, \varepsilon]$ $\rightarrow V[\alpha]$ and $\bar{r}[\alpha, \varepsilon]: D[\alpha, \varepsilon] \rightarrow V[\alpha]$ in the obvious way, by conjugating $r\langle\varepsilon\rangle$ and $\bar{r}\langle\varepsilon\rangle$ with $g[\alpha]$. If $\alpha=[a, b, c, d]$, it will always be assumed when $B[\alpha, \varepsilon]$ or $D[\alpha, \varepsilon]$ is written that $\varepsilon<\min \{b, d\}$. This insures that $D[\alpha, \varepsilon]-V[\alpha]$ lies in the interior of $V[\alpha]$, as does $B[\alpha, \varepsilon]-V[\alpha]$. This will be referred to as the "concavity restriction on $\varepsilon "$ (see Fig. 4).

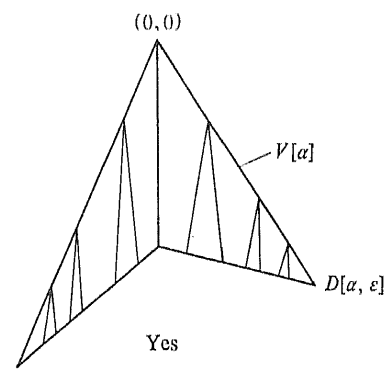

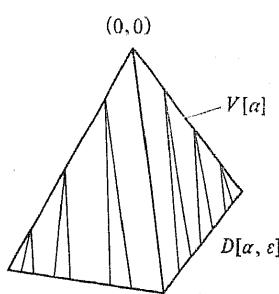

No
Fig. 4. Concavity restriction on 8

Observe that vondition (ii), above, can be restated for $B[\alpha, \varepsilon]$ as follows:

(iii) The accessible points of $B[\alpha, \varepsilon]$ are its endpoints, the points of $V[\alpha]$, and the points of its $v$-exposed $V$-arcs. The only points of $V[\alpha]$ which are accessible in $B[\alpha, \varepsilon]$ by an arc lying entirely in $H(V[\alpha])$ are the endpoints of $V[\alpha]$ and the vertices of the $v$-exposed $V$-arcs of $B[\alpha, \varepsilon]$. In particular, $(0,0)$ is not accessible by such an arc.

Observe also:

(iv) The convex hulls of the $v$-exposed $V$-arcs in $B[\alpha, \varepsilon]$ are pairwise disjoint.

(v) If $v$ is the vertex of a $v$-exposed $V$-arc in $B[\alpha, \varepsilon]$ and $A$ is an arc from $E^{2}-B[\alpha, \varepsilon]$ to $v$ such that $A \subseteq H(V[\alpha])$, then $A \cap I(V) \neq \varnothing$. (This is clear from Figure 4.)

Suppose now that the dendroid $M_{n}$ has been constructed such that $M_{n}$ has no accessible points except its endpoints and the points of countably many exposed $V$-arcs, $\{V\langle n, m\rangle\}_{m=1}^{\infty}$, whose convex hulls are pairwise disjoint.

For each $V\langle n, m\rangle$, choose an $\alpha(n, m)$ such that $V[\alpha(n, m)]$ is isometric with $V\langle n, m\rangle$, and choose an $\varepsilon(n, m)>0$, satisfying the concavity restriction, such that for all $p \in V[\alpha(n, m)]$,

(vi) $\operatorname{dia}\left((\bar{r}[\alpha(n, m), \varepsilon(n, m)])^{-1}(p)\right)<\min \{1 / n, 1 / m\}$.

Then the same inequality is true if $\bar{r}$ is replaced by $r$. 
Let $G\langle n, m\rangle: D[\alpha(n, m), \varepsilon(n, m)] \rightarrow E^{2}$ be an isometric embedding such that $G\langle n, m\rangle(V[\alpha(n, m)])=V\langle n, m\rangle$, and set

and

$$
D\langle n, m\rangle=G\langle n, m\rangle(D[\alpha(n, m), \varepsilon(n, m)])
$$

$$
B\langle n, m\rangle=G\langle n, m\rangle(B[\alpha(n, m), \varepsilon(n, m)]) .
$$

(This "fills in" part of $I(V\langle n, m\rangle)$.) Define $\bar{r}\langle n, m\rangle$ and $r\langle n, m\rangle$ in the obvious way, conjugating through $G\langle n, m\rangle$. Then define:

$$
M_{n+1}=M_{n} \cup\left[\bigcup_{m=1}^{\infty} B\langle n, m\rangle\right]
$$

It is also necessary to define the sequence of continua $\left\{N_{i}\right\}_{i=1}^{\infty}$ by: $N_{1}=\operatorname{Ir}\left(M_{1}\right)$ and $N_{n+1}=M_{n} \cup\left[\bigcup_{m=1}^{\infty} D\langle n, m\rangle\right]$. Then define functions $h_{n}: N_{n+1} \rightarrow M_{n}$ and $h_{n}: M_{n+1} \rightarrow M_{n}$ by:

$$
\begin{aligned}
& \bar{h}_{n} \mid M_{n} \text { is the identity, } \\
& \bar{h}_{n} \mid D\langle n, m\rangle=\bar{r}\langle n, m\rangle \text { and } \\
& h_{n}=\bar{h}_{n} \mid M_{n+1} .
\end{aligned}
$$

Observe that $h_{n} \mid B\langle n, m\rangle=r\langle n, m\rangle$ for each $m$.

The verification that $M_{n+1}$ satisfies the induction hypotheses imposed on $M_{n}$ is relatively straightforward. $M_{n+1}$ (also $N_{n+1}$ ) is compact since if $\varepsilon>0$ only finitely many of the $B\langle n, m\rangle$ 's (also $D\langle n, m\rangle$ 's) contain points at a distance greater than $\varepsilon$ from $M_{n}$ by (vi), above. $M_{n+1}$ is clearly a dendroid, and it is tedious but easy to check, using (iii), (iv), and (v), above, applied (with minor notational alterations) to the $B\langle n, m\rangle$ 's, that the $v$-exposed $V$-arcs of the $B\langle n, m\rangle$ 's are exposed $V$-arcs of $M_{n+1}$ and that the set of accessible points of $M_{n+1}$ consists of these $V$-arcs together with the endpoints of $M_{n+1}$ (see Fig. 5).

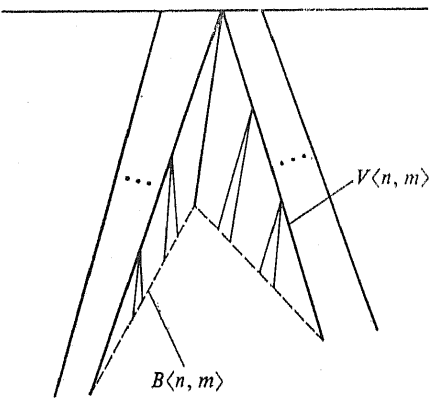

Fig. 5. Small section of $M_{n+1}$, showing $B\langle n, m\rangle$ attached to $V\langle n, m\rangle$
The example, $M$, then, is just the inverse limit of $\left\{M_{i} ; h_{i}\right\}_{i=1}^{\infty}$. To prove that it is planar, it is necessary to verify the hypotheses of Lemma 3 , the AndersonChoquet Theorem.

III. The Anderson-Choquet hypotheses. The following lemmas are relatively easy observations about the collections $\left\{M_{i}\right\}_{i=1}^{\infty},\left\{N_{i}\right\}_{i=1}^{\infty},\left\{h_{i}\right\}_{i=1}^{\infty}$, and $\left\{\bar{h}_{i}\right\}_{i=1}^{\infty}$. The proofs of the less obvious ones are given or indicated.

LEMMA 4. Each $N_{i}$ is the union of $M_{i}$ with the convex hulls of its exposed $V$-arcs.

LemMA 5. Each $h_{n}$ (respectively $h_{n}$ is a continuous monotone retraction of $M_{n+1}$ (respectively $N_{n+1}$ ) onto $M_{n}$.

Proof. The continuity follows easily from (vi), above, and a convergent sequence argument. Once the continuity has been established, it is self-evident that $h_{n}$ (respectively $\bar{h}_{n}$ ) is a monotone retraction since each $r\langle n, m\rangle$ (respectively $\bar{r}\langle n, m\rangle$ ) is.

LemMa 6. Every endpoint of $M_{n}$ is also an endpoint of $M_{i}$ for all $i \geqslant n$.

LemMa 7. For each $n, N_{n+1} \subseteq N_{n} ; M_{n} \subseteq N_{n}$; and $M_{n} \subseteq M_{n+1}$.

Proof. $\quad N_{n+1}=M_{n} \cup\left[\bigcup_{m=1}^{\infty} D\langle n, m\rangle\right]$. However, $D\langle n, m\rangle \subseteq H(V\langle n, m\rangle)$. Thus the first statement follows from Lemma 4 . The other two statements are obvious.

LEMMA 8. If $n$ and $k$ are positive integers, $M_{n} \subseteq N_{k}$.

Proof. Immediate from Lemma 7.

LEMMA 9. $\mathrm{Cl}\left[\bigcup_{n=1}^{\infty} M_{n}\right]=\bigcap_{k=1}^{\infty} N_{k}$.

Proof. $\mathrm{Cl}\left[\bigcup_{n=1}^{\infty} M_{n}\right] \subseteq \bigcap_{k=1}^{\infty} N_{k}$ as a consequence of Lemma 8. Conversely, if $x \in N_{k+1}, d\left(x, M_{k}\right)<k^{-1}$. Thus, if $x \in \bigcap_{k=1}^{\infty} N_{k}, d\left[x, \bigcup_{n=1}^{\infty} M_{n}\right]=0$.

LEMma 10. If $x \in M_{k}-M_{j}$, then $x$ lies in the interior of one of the exposed $V$-arcs in $M_{j}$.

Proof. $x \in M_{k} \subseteq N_{j+1}=M_{j} \cup\left[\bigcup_{i=1}^{\infty} D\langle j, i\rangle\right]$, but $x \notin M_{j}$, so $x \in D\langle j, i\rangle$ for some $i$. $x \notin V\langle j, i\rangle$, so by the concavity restriction, $x \in D\langle j, i\rangle-V\langle i, j\rangle$ $\subseteq I(V\langle j, i\rangle)$.

LEMMA 11. $h_{n}$ is constant on the convex hull of each exposed V-arc in $M_{n+1}$.

Proof. Each $\bar{r}\langle n, m\rangle$ is constant on the convex hull of each exposed $V$-arc in $M_{n+1}$ where it is defined.

LEMMA 12. If $V$ is an exposed $V$-arc in $M_{k}$ and $1 \leqslant n \leqslant k, V$ lies in (and hence cilso $H(V)$ ) the convex hull of some $L$, where $L$ is an exposed $V$-arc of $M_{n}$.

LEMMA 13. If $V$ is an exposed $V$-arc in $M_{k}$ and $1 \leqslant n<k, h_{n}$ is constant on $H(V)$. 
Proof. This is immediate from Lemmas 11 and 12.

Recall the notation $h[n, k]$, above

LEMMA 14. If $k \geqslant n, h[k, n]=\bar{h}_{n} \mid M_{k}$.

Proof. For $k=n$, both these functions are the identity on $M_{k}$. For $k=n+1$, $h[n+1, n]=h_{n}$, and $h_{n}=\bar{h}_{n} \mid M_{n+1}$ by definition. Suppose the lemma be established for some value $k \geqslant n+1$. Then if $x \in M_{k+1}$, cither $x \in M_{k}$ or $x \notin M_{k}$. If $x \in M_{k}$, $h[k+1, n](x)=h[k, n] \circ h_{k}(x)=h[k, n](x)=h_{n}(x)$ by the induction hypothesis. If $x \notin M_{k}, h[k+1, n](x)=h\lceil k, n] \circ h_{k}(x)=\Gamma_{n}\left(h_{k}(x)\right)$ by the induction hypothesis. However, $h_{k}(x)$ and $x$ both lie in $H(V\langle k, m\rangle)$ for some $m$, and by Lemma 13, since $n<k, \bar{h}_{n}$ is constant on $H(V\langle k, m\rangle)$. Thus, $h_{n}\left(h_{k}(x)\right)=h_{n}(x)=h[k+1, n](x)$, and $h[k+1, n](x)=h_{n}\left(h_{k}(x)\right)=h_{n}(x)$, and the proof is complete.

LeMma 15. If $x \in M_{n}$, dia $\left[\bigcup_{k=n}^{\infty}(h[k, n])^{-1}(x)\right]<1 / n$.

Proof. By Lemma $14,(h[k, n])^{-1}(x) \subseteq\left(h_{n}\right)^{-1}(x)$ for all $k>n$. Thus,

$$
\operatorname{dia} \bigcup_{k=n}^{\infty}(h[k, n])^{-1}(x) \leqslant \operatorname{dia}\left(h_{n}\right)^{-1}(x)<1 / n .
$$

Lemma 16. For any $n$, the collection $\{h[k, n] \mid k \geqslant n\}$ is an equi-uniformly continuous family.

Proof. By Lemma 14, all are restrictions of the same uniformly continuous function, $\bar{h}_{n}$.

Lemmas 15 and 16 given us precisely the hypotheses of the Anderson-Choquet theorem, our Lemma 3. Hence $M$ is topologically the closure of the union of the $M_{i}$ s.

IV. The properties of $M$. We have three different representations of $M$, one as the inverse limit of the sequence $\left\{M_{i} ; h_{i}\right\}$, another as $\mathrm{Cl}\left[\bigcup_{n=1}^{\infty} M_{n}\right]$, and another as $\bigcap_{n=1}^{\infty} N_{n}$. The relationship between the first two of these, given in Lemma 3 , is used strongly here. The inverse limit $M$, will now be identified with $\mathrm{Cl}\left[\bigcup_{i=1}^{\infty} M_{i}\right]$ to simplify the terminology.

LEMMA 17. If $x$ is an endpoint of some $M_{k}, x$ is an endpoint of $M$.

Proof. This is an easy consequence of the fact that no arc is extended beyond its original endpoints in the construction of $M_{n+1}$ from $M_{n}$.

THEOREM 1. No nonendpoint of $M$ is arcwise accessible in $M$.

Proof. A nonendpoint, $x$, of $M$ lies in $M_{\infty}$ and hence in some $M_{k}$, by Lemma 2. Then $x$ is a nonendpoint of $M_{k}$ by Lemma 17 , and

$$
M_{k} \subseteq M_{k+1} \subseteq M_{k+2} \subseteq M \text {. }
$$

The reader can easily check that no nonendpoint of $M_{k}$ is accessible in $M_{k+2}$, so that $x$ is not accessible in $M_{k+2}$ and hence not in $M$.

Before proceeding to the proof that each endpoint of $M$ is accessible in $M$, the following technical lemma will be proven.

Lemma 18. If $\left\langle x_{i}\right\rangle_{i=1}^{\infty} \in M-M_{\infty}$, then for each $i, x_{i+1} \neq x_{i}$, and $x_{i}$ is the vertex of one of the exposed V-arcs in $M_{i+1}$.

Proof. Suppose for some $i, x_{i}=x_{i+1}$. Since $\left\langle x_{i}\right\rangle_{i=1}^{\infty}$ is not eventually constant, there is a smallest $k>i$ such that $x_{k} \neq x_{i}$. We have $k \geqslant i+2$. Then since $x_{k-1}=x_{i}$, $x_{k-1} \neq x_{k}$, and this means that $x_{k-1}$ lies on one of the exposed $V$-arcs in $M_{k-1}$, since no other points have non-degenerate preimages under $h_{k-1}$. If $k-1>i+1$, this exposed $V$-arc contains no point of $M_{i}$, which is impossible since $x_{k-1}=x_{i} \in M_{i}$. Thus $k-1 \leqslant i+1$, or $k \leqslant i+2$. Since $k \geqslant i+2$, we nust have $k=i+2$. Thus $x_{i}=x_{i+1}$. and $x_{i+1} \neq x_{i+2}$. Since $x_{i+1}$ must lie on one of the exposed $V$-arcs in $M_{i+1}$, and must belong to $M_{t}, x_{i+1}$ is a vertex of one of the exposed $V$-arcs of $M_{i+1}$. But then $x_{i+1}$ is not the vertex of a $V$-arc in $M_{i+2}$, and $\left(h_{i+1}\right)^{-1}\left(x_{i+1}\right)$ is a segment. Thus $x_{i+2}$ does not lie on an exposed $V$-arc of $M_{i+2}$, and none of the exposed $V$-arcs of $M_{j}$ for $j \geqslant i+2$, can contain $x_{i+2}$, so that for $j \geqslant i+2, x_{i+2}=x_{j}$, and the sequence is eventually constant and so not in $M-M_{\infty}$. Thus for each $i, x_{i} \neq x_{i+1}$.

Since for any $i, x_{i+1}$ must lie on an exposed $V$-arc of $M_{i+1}$, and $h_{i}\left(x_{i+1}\right)$ is the vertex of that $V$-arc, $x_{i}$ is the vertex of an exposed $V$-arc in $M_{i+1}$.

THEOREM 2. Every endpoint of $M$ in arcwise accessible in $M$.

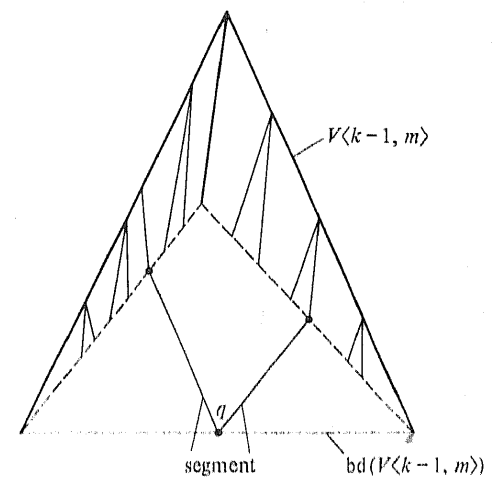

Fig. 6

Proof. If $x$ is an endpoint of some $M_{k}$, let $k$ be the smallest integer such that $x \in M_{k}$. If $k=1$, a vertical segment to $x$ from above or below shows that $x$ is accessible in $N_{1}$ and hence in $M \subseteq N_{1}$. If $k>1, x$ is an endpoint of $B\langle k-1, m\rangle$ for some $m$. Let $q$ be a point on bd $(V\langle k-1, m\rangle)$. The straight line segment from $q$ to $x$ misses $N_{k}-\{x\}$, so that $x$ is accessible in $N_{k}$ and hence in $M$ since $M \subseteq N_{k}$ (see Fig. 6). 4 - Fundarnenta Mathematicac $\mathrm{CX} / 3$ 
Suppose then that $x \in M-M_{\infty}$ (Figure 7 may be helpful here). Then $x=\left\langle x_{i}\right\rangle_{i=1}^{\infty}$ where $x_{i} \neq x_{i+1}$ for all $i$, and each $x_{i}$ is a vertex of an exposed $V$-arc, $V_{i}$, in $M_{i+1}$. Let $V_{0}$ be the exposed $V$-arc in $M_{1}$ on which $x_{1}$ lies, and let $q_{i}$ denote the midpoint of $\operatorname{Bd}\left(V_{i}\right)$ for each $i \geqslant 0$. Then, for $i>1, q_{i}$ and $x_{i}$ both belong to $\left(\bar{h}_{i}\right)^{-1}\left(x_{i}\right)$, so that

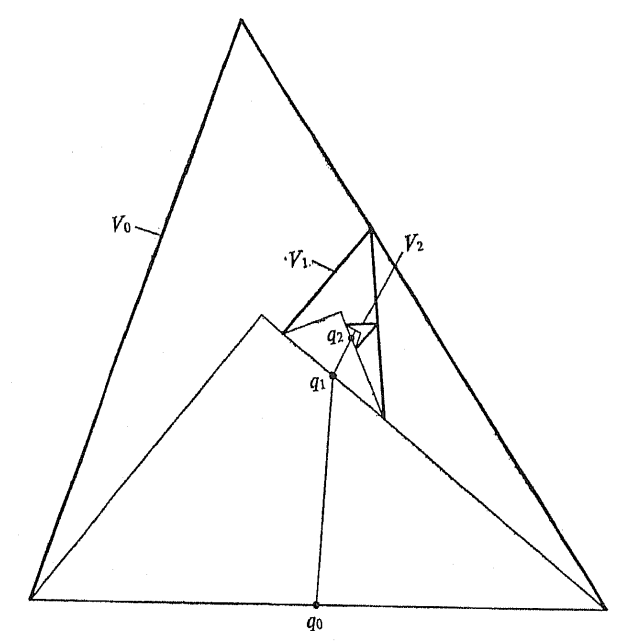

Fig. 7

$d\left(q_{i}, x_{i}\right)<i^{-1}$. Then since $\left\langle x_{i}\right\rangle$ converges to $x,\left\langle q_{i}\right\rangle$ converges to $x$ also. The straight line segment $\left[q_{i}, q_{i+1}\right]$ misses $N_{i+2}$ and hence misses $M$. Then, the closure of $\bigcup^{\infty}\left[q_{i}\right.$,

$\bigcup_{i=0}^{\infty}\left[q_{i}, q_{i+1}\right]$ is an arc from $q_{0}$ to $x$ missing $M$ except for the point $x$, so that $x$ is accessible in $M$.

$V$. The connectedness properties of the set of endpoints of $M$. The next lemma is a fairly simple generalization of Theorem 6 of [2, p. 129]. I am indepted to J. Krasinkiewicz for calling it to my attention.

LEMMA 19. If $S$ is a connected one-manifold and $E$ is a set contained in any Euclidean space and $f: S \rightarrow E$ is surjection of Baire class I such that for any open interval $(a, b) \subseteq S,\{a, b\} \subseteq \mathrm{Cl}(f(a, b))$, then $E$ is connected.

The following technical set - theoretic lemma is needed in the proof of the main result of this section.

Lemma 20. Suppose $\left\{A_{i}\right\}_{i=1}^{\infty}$ is a countable collection of pairwise disjoint countable sets, and suppose that $g: \bigcup_{i=2}^{\infty} A_{i} \rightarrow \bigcup_{i=1}^{\infty} A_{i}$ is a function such that $g\left(A_{i+1}\right) \subseteq A_{i}$ 
Then $\mathrm{Bd}\left(V_{n}\right)$ is mapped piecewise linearly onto $U_{n}$, the midpoint going to the vertex, and otherwise $f_{n}(S)$ is undisturbed. $f_{n+1}$ is defined to be $e_{n} \circ f_{n}$. (Referring to Figure 8 may be helpful.) Then each $f_{n}$ is continuous and one-to-one. Now let $p \in S$. If for some $n, f_{n}(p)$ is an endpoint of some $M_{k}$, it follows that for all $m>n$, $f_{m}(p)=f_{n}(p)$, so that $\lim f_{m}(p)=f_{n}(p) \in E$.

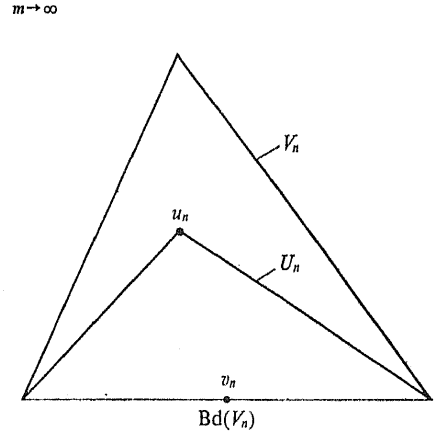

Fig. 8

If $f_{n}(p)$ is not an endpoint of any $M_{k}$ for any $n$, then for each $n$ there is a unique $k_{n}$ such that $f_{n}(p) \in \operatorname{Bd}\left[V_{k_{n}}\right]$. We have $k_{n} \geqslant n$, and if $k_{n}>n$, then $k_{n+1}=k_{n}$. Thus, for infinitely many $n, k_{n}=n$. Let $\left\langle n_{i}\right\rangle_{i=1}^{\infty}$ be the increasing sequence of those and only those $n$ for which $k_{n}=n$. Then $f_{n_{i}}(p) \in \mathrm{Bd}\left[v_{n_{l}}\right]$, so that $f_{1+n_{l}}(p) \in U_{n_{l}}$, and $f_{1+n_{i}}(p) \in \operatorname{Bd}\left[V_{k_{1+n_{i}}}\right] \subseteq U_{n_{i}}$. Furthermore

$$
\operatorname{dia}\left[f_{1+n_{i}}^{-1}\left(\operatorname{Bd}\left(V_{k_{1+n_{t}}}\right)\right)\right]<\frac{1}{2} \operatorname{dia} f_{n_{t}}^{-1}\left(\operatorname{Bd}\left(V_{m}\right)\right)
$$

for each $i$, so that $p$ is the only point in $\bigcap_{n=1}^{\infty} f_{n}^{-1}\left(\operatorname{Bd}\left(V_{k_{n}}\right)\right)$. Let $\left\langle x_{i}\right\rangle_{i=1}^{\infty}$ be the point in $M$ such that $x_{i}$ is the vertex of $V_{m+1}$ for each $i$. (Observe that $V_{n_{i+1}}$ is an exposed $V$-arc of $M_{i+1}$ for each $i$ and that there is such a point $\left\langle x_{i}\right\rangle_{i=1}^{\infty}$. (Refer to Figure 9.)

Then, for $i>1, d\left(x_{i}, f_{1+n_{i}}(p)\right)<1 /(i-1)$, since both belong to $h_{i-1}^{-1}\left(x_{i-1}\right)$. Thus $\left\langle f_{n_{i}}(p)\right\rangle_{i=1}^{\infty}$ converges to $\lim _{i \rightarrow \infty} x_{i}$, which is an endpoint of $M$. Furthermore, if $q \in S$ and $q \neq p$, then for some $i, q \notin f_{n_{i}}^{-1}\left(\operatorname{Bd}\left(V_{m}\right)\right)$ so that $\lim _{n \rightarrow \infty} f_{n}(p) \neq \lim _{n \rightarrow \infty} f_{n}(q)$. Thus, if $f: S \rightarrow E^{2}$ is defined by $f(x)=\lim _{n \rightarrow \infty} f_{n}(x), f$ is one-to-one and $f(S) \subseteq E$. However, if $x \in E$, either $x \in M_{k}$ for some $k$ or $x \in M-M_{\infty}$. If $x \in M_{k}$ for some $k$, either $k=1$ or $k \neq 1$. If $k=1, x \in S$ and $f_{n}(x)=x$ for each $n$ so that $f(x)=x$. If $k>1, x$ lies on $U\langle k-1, m\rangle$ for some $m$ and $U\langle k-1, m\rangle=U_{J}$ for some $j$. Let $y=f_{j+1}^{-1}(x) ; y \in S$, and for all $k \geqslant j+1, f_{k}(y)=x$, so that $f(y)=x$. In case $x \in M-M_{\infty}$ there is a unique subsequence $\left\langle V_{n_{i}}\right\rangle_{i=1}^{\infty}$ of $\left\langle V_{n}\right\rangle_{n=1}^{\infty}$ such that: 1) $V_{n}$ is an exposed $V$-arc of $\left.M_{i}, 2\right) V_{n_{i+1}} \subseteq H\left(V_{n}\right)$; and 3) $x \in H\left(V_{n_{i}}\right)$ for each $i$. Then, by 
to $(0,1)$; and $F$ - is the union of all segments from $(0,-2)$ to $(1 / n,-1)$ for $n$ even and the segment from $(0,-2)$ to $(0,-1)$. For $n \neq 0$, let

$$
\begin{aligned}
& a_{n}=\left\{\begin{array}{lll}
\left(\frac{1}{2 n-1},-1\right), & \text { if } & n>0, \\
\left(\frac{1}{2 n+1},-1\right), & \text { if } & n<0,
\end{array}\right. \\
& b_{n}=\left(\frac{1}{2 n}, 1\right) .
\end{aligned}
$$

$W$ is now obtained from $W_{0}$ by attaching a homeomorphic copy of $K$ along its edge to each of the arcs: $\left[a_{-1}, a_{1}\right],\left[a_{n}, a_{n+1}\right],\left[b_{-1}, b_{1}\right]$ and $\left[b_{n}, b_{n+1}\right]$ in $W_{0}$ on the side such that this is possible, and such that: 1) these copies of $K$ are disjoint except where they necessarily meet along their edges; 2) these copies of $K$ meet $W_{0}$ only along their edge; and 3) for any $\varepsilon>0$ only finitely many of these copies of $K$ contain points at a distance greater than $\varepsilon$ from $W_{0}$. The pattern can be seen in Figure 10, where the attached copies of $K$ are indicated $(K)$. The idea is to "cove up" all accessible non-endpoints of $W_{0} . W$ is the union of $W_{0}$ and the countably

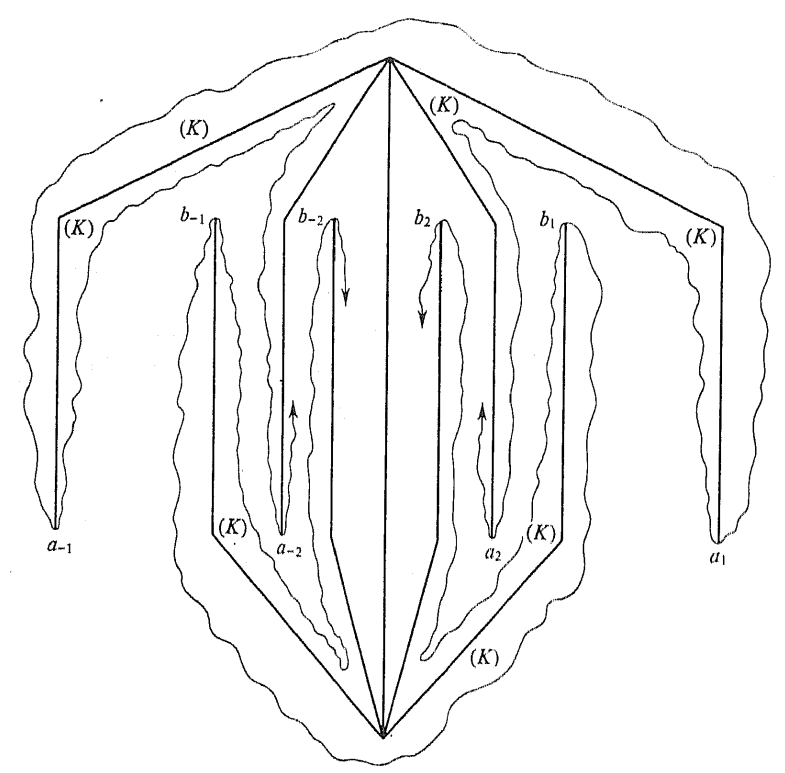

Fig. 10. $W_{0}$ is pictured in heavy lines, the remainder of the construction is pictured schematically in thinner lines many attached copies of $K . W$ is a dendroid and the endpoints and accessible points of $W$ are the same; however, the set of endpoints of $W$ is not connected since $W$ is separated by the segment $\{0\} \times[-2,2]$, which contains no accessible points, and this yields a separation of the set of accessible points of $W$

Next, to show that $M$ is not irreducible with respect to its stated properties, define a sequence $\left\langle M_{i}^{\prime}\right\rangle_{i=1}^{\infty}$, where $M_{i}^{\prime}$ is a subcontinuum of $M_{i}$ for each $i$ such that $h_{i}$ maps $M_{i+1}^{\prime}$ to $M_{i}^{\prime}$ in such a way that the arguments concerning the properties of $M$ can be repeated. This can be done as follows:

Let $M_{1}^{\prime}=\left\{(x, y) \in M_{1} \mid y \geqslant-\frac{10}{19}\right\}$ (The value $-\frac{10}{19}$ is chosen here only so that the line used to truncate $M_{1}$ does meet bd( $\left.V\right)$ for any exposed $V$-arc $V$. It does meet the "outer" endpoints of the two broadest exposed $V$-arcs in $M_{1}$ (see Fig. 2).

If $M_{n}^{\prime}$ has been defined, consider any exposed $V$-arc $V$ in $M_{n}$. It will be a subarc of an exposed $V$-arc $V\langle n, m\rangle$ in $M_{n}$. In order to define $M_{n+1}^{\prime}$, simply attach a portion

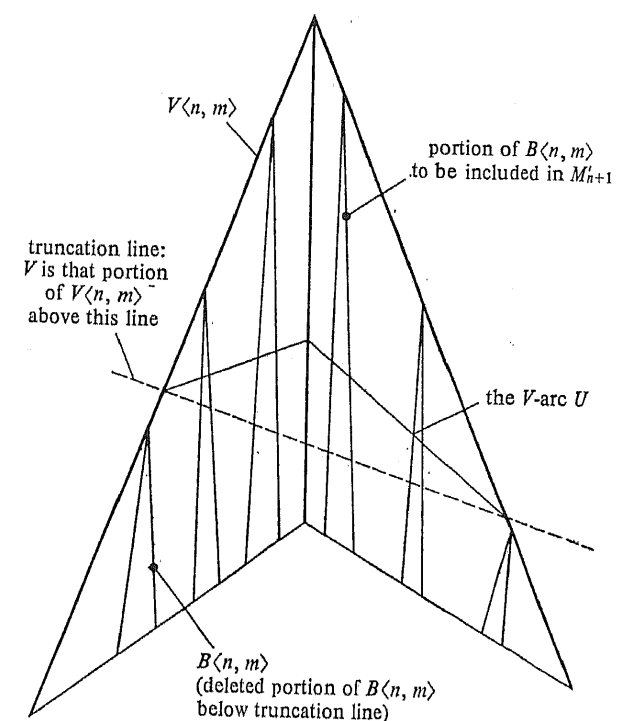

Fig. 11. The $V$-arc $U$ becomes in each case the truncation line for the $V$-arcs at the next stage of the construction. If the $V-\operatorname{arc} V$ is all of $V\langle n, m\rangle$, then all of $B\langle n, m\rangle$ is included in $M_{n+1}$

of each $B\langle n, m\rangle$, determined by truncating $B\langle n, m\rangle$ with a $V$-arc $U$ with the same endpoints as $V$ and lying in $H(V)$ and having its vertex on the $\operatorname{arc}$ in $B\langle n, m\rangle$ from the vertex of $V$ (see Fig. 11).

When this truncation and cutting out of part of $B\langle n, m\rangle$ has been done for each exposed $V$-arc of $M_{n}^{\prime}$, then $M_{n+1}^{\prime}$ is the union of $M_{n}^{\prime}$ and the remaining portion 
of the $B\langle n, m\rangle$ 's. (Of course, some of the exposed $V$-arcs of $M_{n+1}$ are omitted completely from $M_{n+1}^{\prime}$, as can be seen from the deleted portions of $B\langle n, m\rangle$ in Figure 11.)

It is easy to see that all of the argument for the $M_{n}$ 's can be carried out for the $M_{n}^{\prime}$ s, defining $N_{n}^{\prime}$ to be the union of $M_{n}^{\prime}$ with the convex hulls of all its exposed $V$-arcs. Thus $M^{\prime}$, the inverse limit of $\left\{M_{n}^{\prime} ; h_{n} \mid M_{n+1}^{\prime}\right\}_{n=1}^{\infty}$, is a proper subcontinuum of $M$ with the same connectedness and accessibility properties for its set of endpoints.

\section{References}

[1] R. D. Anderson and G. Choquet, A plane continuum no two of whose nondegenerate subcontinua are homeomorphic: An application of inverse limits, Proc. Amer. Math. Soc. 10 (1959), pp. 347-353.

[2] K. Kuratowski, Topology, Vol. II, New York-London-Warszawa 1968.

[3] A. Lelek, On plane dendroids and their endpoints in the classical sense, Fund. Math. 49 (1961), pp. 301-319.

\section{UNIVERSTTY OF DELAWARE}

Newark Delaware

\section{An example concerning automorphisms of generalized cubes}

\section{by}

\author{
Dorothy Maharam (Rochester, N. Y.)
}

Abstract. An example is given of an involution $T$ on $I^{m}(m \geqslant c)$ with the following properties: (1) $T=T^{-1}$ sends Baire sets to Baire sets, (2) $T$ induces the identity automorphism on both the category algebra and the measure algebra of $I^{m}$, (3) $T$ has no fixed points.

Let $m$ be an infinite cardinal, and consider the product $I^{m}$ of $m$ copies of the unit interval $I$, both as a topological space (with product topology) and as a measure space (with product Lebesgue measure). It is known ([1]) that every automorphism of the measure algebra of $I^{m}$ can be "realized" by a point map that can even be required to be a Baire isomorphism; and a sinilar remark applies to the category algebra ([5]). Some time ago, S. Kakutani asked the author whether the realization would have to be "almost" unique. More specifically, suppose $T$ is a transformation of $I^{m}$ that induces the identity automorphism of the measure algebra (or of the category algebra) of $I^{m}$; need $T(x)=x$ for a "large" set of $x$ 's? When $m \leqslant s_{0}$ the answer is easily seen to be "yes"; we have only to discard, for each member of a countable base $U_{1}, U_{2}, \ldots$, for $I^{m}$, the negligible set $U_{n} \Delta T\left(U_{n}\right)$, and $T$ will be the identity transformation on what is left. But we show here that, for $m \geqslant c$, the answer is "no" to both the measure and category forms of the question, and by the same example even if the transformation is required to be an involution. It is of course enough to consider $m=c$.

THeorem. There exists a Baire isomorphism $T$ of $I^{c}$, of period 2 , such that $T$ induces the identity automorphisms of both the category algebra and the measure algebra of $I^{c}$, but such that $T$ has no fixed points $\left({ }^{1}\right)$.

Proof. We use the following notation. $I$ denotes the closed unit interval $[0,1]$ as usual; $J=I \cup\{-1\}, X=I^{c}$ regarded as $\prod\left\{I_{j}: j \in J\right\}$, where each $I_{j}=I$. The $j$ th coordinate of $x \in X$ is $\pi_{j}(x)$ or $x(j)$. (As this notation suggests, the coordinate $x(-1)$ will play an exceptional role).

Take an arbitrary measure-and-category-preserving Borel isomorphism $\xi$ of $I$ onto itself, without fixed points, but such that $\xi^{2}=$ identity map $\iota_{I}$ of $I$. For instance,

(1) For a simple example of such a $T$ for which the set of fixed points is not measurable (having outer measure 1 and inner measure 0 ) see [3, p. 702]. 